CLINICAL STUDY

\title{
High prevalence of hirsutism and menstrual disorders in obese adolescent girls and adolescent girls with type 1 diabetes mellitus despite different hormonal profiles
}

\author{
Dinane Samara-Boustani $^{1, *}$, Ana Colmenares ${ }^{1, *}$, Caroline Elie $^{2}$, Myriam Dabbas $^{3}$, Jacques Beltrand ${ }^{1}$, \\ Virgile Caron $^{1}$, Claude Ricour ${ }^{3}$, Paul Jacquin ${ }^{4}$, Nadia Tubiana-Rufi ${ }^{4}$, Claire Levy-Marchal ${ }^{4}$, Christine Delcroix ${ }^{4}$, \\ Delphine Martin $^{1}$, Lila Benadjaoud ${ }^{5}$, Evelyne Jacqz Aigrain ${ }^{5}$, Christine Trivin ${ }^{6}$, Kathleen Laborde ${ }^{6}$, \\ Elisabeth Thibaud ${ }^{1}$, Jean-Jacques Robert ${ }^{1}$ and Michel Polak ${ }^{1}$ \\ ${ }^{1}$ Paediatric Endocrinology and Diabetes Unit, ${ }^{2}$ Biostatistics Department and ${ }^{3}$ Obesity and Nutrition Paediatric Unit, Assistance Publique - Hôpitaux de \\ Paris, Université Paris Descartes, Hôpital Necker-Enfants Malades, 149 rue de Sèvres, 75015 Paris, France, ${ }^{4}$ Paediatric Endocrinology and Diabetes Unit \\ and ${ }^{5}$ Clinical Investigation Center, Assistance Publique - Hôpitaux de Paris, Hôpital Robert Debré, Paris, France and ${ }^{6}$ Functional Testing Unit, Assistance \\ Publique - Hôpitaux de Paris, Hôpital Necker-Enfants Malades, Paris, France \\ (Correspondence should be addressed to M Polak; Email: michel.polak@nck.aphp.fr)
}

*(D Samara-Boustani and A Colmenares contributed equally to this work and should be considered as co-first authors)

\begin{abstract}
Objectives: To compare the pubertal development, the hormonal profiles and the prevalence of hirsutism and menstrual disorders in obese adolescent girls and adolescent girls with type 1 diabetes mellitus (T1DM).

Methods: Data were collected from 96 obese adolescent girls and 78 adolescent girls with T1DM at Tanner stage IV or V, whose ages ranged between 11.9 and 17.9 years.

Results: High prevalence of hirsutism and menstrual disorder was found in the obese adolescent girls ( 36.5 and $42 \%$ respectively) and the adolescent girls with T1DM ( 21 and $44 \%$ respectively). The obese girls were significantly younger at pubarche, thelarche and menarche than the girls with T1DM. Hirsutism in the obese girls and those with T1DM was associated with hyperandrogenaemia and a raised free androgen index (FAI). When the cause of the raised FAI was investigated in both the groups of girls with hirsutism, the raised FAI in the obese girls was due to low serum sex hormone-binding globulin (SHBG) levels. In contrast, the raised FAI of the girls with T1DM and hirsutism was due to hyperandrogenaemia. Menstrual disorders in the T1DM girls were associated also with hyperandrogenaemia unlike obese girls.

Conclusions: Hirsutism and menstrual disorders are common in obese adolescent girls and adolescent girls with T1DM. Although hyperandrogenaemia is present in both groups of girls, the androgenic profiles of the two groups differ. The hyperandrogenaemia in the obese girls is primarily due to their decreased serum SHBG levels, whereas the hyperandrogenaemia in the girls with T1DM is due to their increased androgen production.
\end{abstract}

European Journal of Endocrinology 166 307-316

\section{Introduction}

An increased prevalence of hyperandrogenism has been described in obese adolescent girls and adult women and in those with type 1 diabetes mellitus (T1DM). In obese girls and women, hyperandrogenism has been related to their insulin resistance and hyperinsulinaemia $(1,2,3)$. In women with T1DM, the aetiology of their hyperandrogenism is unclear, and it has been proposed that their hyperandrogenism is due to non-physiological insulin replacement therapy $(4,5)$. Indeed, the results of several laboratory and clinical studies have found that insulin can also influence the production of ovarian and adrenal androgens $(6,7,8,9,10,11)$.
Since hirsutism and menstrual disturbances are two clinical phenomena that are usually secondary to hyperandrogenaemia, the prevalence of hirsutism and menstrual disorders in obese women and those with T1DM would be expected to be higher than those found in the general female population $(12,13,14,15,16)$. In women with T1DM, menstrual disorders have been associated with poor glycaemic control $(15,17,18)$, weight gain (4) and high-insulin doses (15). However, Escobar-Morreale et al. (5) did not find a significant difference in glycaemic control and insulin doses in hyperandrogenic and nonhyperandrogenic women with T1DM.

Obese adolescent girls and those with T1DM share a number of gynaecological characteristics and 
pathophysiological events. The prevalence of hirsutism and its hormonal profile has been investigated only in adult women $(19,20)$ and is unknown in obese adolescent girls and adolescent girls with T1DM. To the best of our knowledge, a systematic comparison on the pubertal development, the prevalence of hirsutism and menstrual disorders and the hormonal profile of obese adolescent girls and adolescent girls with T1DM has not been done. Hence, the objectives of our investigation were: i) to first characterise the clinical characteristics and the serum hormone profiles of obese adolescent girls and adolescent girls with T1DM with either hirsutism or a menstrual disorder; and ii) to compare the prevalence, pubertal development and hormonal profiles of hirsutism and menstrual disorders in these two groups of adolescent girls.

\section{Subjects and methods}

\section{Subjects}

This was a cross-sectional study in which we compared the pubertal development, hormonal profiles and prevalence of hirsutism and menstrual disorders in 96 obese adolescent girls and 78 adolescent girls with T1DM. The 174 study participants were recruited from patients who presented for routine follow-up visits to the Obesity and Nutrition Unit, Necker Children's Hospital, or the Paediatric Endocrinology and Diabetes Unit, Necker Children's Hospital and Robert Debré Hospital in Paris, France between March 2006 and March 2008. The institutional review board of both the hospitals approved the study, and written informed consent was obtained from all adolescent girls and their parents before study inclusion.

All girls were younger than 18 years and were at Tanner stage IV or V. None of the girls were pregnant, had received sex steroids or any drug that was known to affect ovarian function for at least 6 months before the study, and did not show clinical signs of secondary hyperandrogenism or other pubertal disorders. The girls with T1DM were included in this study if they had been diagnosed with autoimmune T1DM by the presence of $\beta$-cell antibodies in their serum, and had been treated with insulin from the time of its diagnosis. The obese girls were included in the study if their body mass index (BMI) Z-score without any medication was $>2$. Girls were excluded from the study if they suffered from type 2 diabetes mellitus (T2DM) or other types of diabetes mellitus, abnormal thyroid function, or suffered from a genetic disease, Cushing's disease, hyperprolactinaemia or congenital adrenal hyperplasia.

\section{Research strategy}

The study girls were divided into eight cohorts: obese girls with hirsutism, obese girls without hirsutism, girls with T1DM and hirsutism, girls with T1DM and without hirsutism, obese girls with a menstrual disorder, obese girls without a menstrual disorder, girls with T1DM and a menstrual disorder and girls with T1DM without a menstrual disorder. After collecting the data on the clinical characteristics and the serum hormone profiles of each cohort, we first compared the clinical characteristics and the serum hormone profiles of the obese girls with and without hirsutism, and those with and without menstrual disorders. We did the same comparison in the girls with T1DM. We then compared the data from the obese girls with either hirsutism or a menstrual disorder with those from the equivalent girls with T1DM.

\section{Methods}

A standardised history was collected and a physical examination was done on all the study girls by the same physician. The family history (first degree) of T2DM and obesity was recorded in both cohorts. In the obese girls, the duration of obesity was established by reviewing the anthropometric parameters in their medical charts (this information was present in the records of 77 of the 96 girls). For the girls with T1DM, the duration of their diabetes mellitus was established by reviewing their medical records.

For each study girl: height was measured using a Harpenden stadiometer; BMI was calculated from each girl's weight $(\mathrm{kg})$ and height $(\mathrm{m})$ (21) and then expressed as the Z-score according to the French normative data (22); waist circumference was measured at the narrowest point between the lower costal margin and the iliac crest in the standing position; and hip circumference was measured at the widest point near the greater trochanter. The waistto-hip ratio was determined using these last two measurements. The presence of acanthosis in the study cohorts was clinically assessed.

We prospectively categorised each cohort into four groups according to the presence or absence of hirsutism and whether they have a history of menstrual disorders. Hirsutism was considered to be present when the modified Ferriman-Gallwey score was $\geq 8$ (23). Delayed puberty was considered to have occurred by the absence of breast development by the time the girl was 13 years old. Menstrual disorders were assessed only in girls whose gynaecological age was at least 2 years (24, $25,26)$. Data on each girl's pubertal development and menstrual history were obtained retrospectively by interviewing each girl and her mother and by reviewing each girl's medical record. For the menstrual history, each girl was asked about whether she had more or less than eight menstrual cycles per year, the cycle regularity and the duration in days of each cycle during the last 12 months. A menstrual disorder was considered to be present under the following conditions: the presence of primary or secondary amenorrhoea, the complete absence of menstruation by the age of 16 or 4 
years after thelarche, the complete absence of menstruation for three or more months; oligomenorrhoea that is defined as menstruation that occurred at intervals of $>45$ days or fewer than eight times per year; polymenorrhoea that is defined as menstruation that occurred at intervals of $<25$ days; an irregular menstrual cycle that is defined as both oligomenorrhoea and polymenorrhoea occurring during the past 12 months; and menorrhagia that is defined as a menstrual period that lasted longer than 7 days $(27,28)$.

\section{Determination of serum hormone levels}

Fasting blood samples were obtained early in the morning between the third and fifth days of the menstrual cycle in girls with regular menstrual cycles and randomly in girls with irregular menstrual cycles. The following determinations were made in the serum of all study participants: total testosterone (TT), androstenedione (A), DHEAS and sex hormone-binding globulin (SHBG). All androgen and SHBG assays were done in the clinical biochemistry laboratory of the Necker Children's Hospital. Other assays were done by identical methods either in the clinical biochemistry laboratory of the Necker Children's Hospital or that of the Robert Debré Hospital. The serum levels of the androgens were interpreted according to the reference laboratory range for Tanner stage IV or $\mathrm{V}$ girls, namely serum TT levels between 0.12 and $0.59 \mathrm{ng} / \mathrm{ml}$, serum A levels between 0.73 and $2.21 \mathrm{ng} / \mathrm{ml}$, serum DHEAS levels between 240 and $2389 \mathrm{ng} / \mathrm{ml}$ and serum SHBG levels between 30 and $69 \mathrm{nmol} / \mathrm{l}$ (29). The free androgen index (FAI) was calculated using the formula: serum TT level $(\mathrm{nmol} / \mathrm{l}) /$ serum SHBG level $(\mathrm{nmol} / \mathrm{l}) \times$ $100(30)$.

Commercial RIA kits were used to measure serum TT levels (Testo-CT2, CisBio International, Gif sur Yvette, France); serum A levels (Immunotech, Marseille, France) and serum DHEAS levels (Immunotech), in accordance with each manufacturer's instructions after organic solvent extraction and celite column partition chromatography for TT and A. Serum SHBG levels were measured using a commercial RIA (SHBG-RIA CT, CisBio International), according to the manufacturer's instructions. All serum determinations were made in duplicate, and the average of the two measurements was used in the statistical analysis of the data. The sensitivity of the: i) TT assay was $0.03 \mathrm{ng} / \mathrm{ml}$; ii) A assay was $0.04 \mathrm{ng} / \mathrm{ml}$; iii) DHEAS assay was $60 \mathrm{ng} / \mathrm{ml}$; and iv) SHBG assay was $0.5 \mathrm{nmol} / \mathrm{l}$. The inter- and intraassay coefficients of variation for the: i) TT assay were $\leq 7.5$ and $\leq 7.0 \%$ respectively; ii) A assay were $\leq 8.1$ and $\leq 11.9 \%$ respectively; iii) DHEAS assay were $\leq 7.4$ and $\leq 10.5 \%$, respectively; and iv) SHBG assay were $\leq 5.2$ and $\leq 5.5 \%$ respectively.

The obese adolescent girls underwent an oral glucose tolerance test (OGTT) with measurements of blood glucose and plasma insulin levels at 0,30 and $120 \mathrm{~min}$. Serum HbA1c levels were also measured in these girls. Their insulin release was determined by the homeostasis model assessment of insulin resistance (HOMA-IR) and calculated using the formula: fasting blood glucose levels $(\mathrm{mmol} / \mathrm{l}) \times$ fasting plasma insulin level $(\mathrm{IU} / \mathrm{ml}) / 22.5$. Their insulin sensitivity was determined by the homeostatic model assessment for $\beta$-cell function (HOMA\%B) and calculated using the following formula: $20 \times$ fasting plasma insulin level/fasting blood glucose level-3.5 $(31,32,33)$. Blood glucose levels were determined colorimetrically (Hitachi 977, Hitachi Ltd.). Plasma insulin levels were determined using a radioimmunometric sandwich assay (Bi-Insulin IRMA, CisBio International). Serum HbA1c levels were determined by HPLC (Tosoh Bioscience, Tokyo, Japan).

\section{Statistical analysis}

Data for normally distributed continuous variables are presented as the mean \pm S.D. and data for non-normally distributed continuous variables as the median (range). Differences in categorical variables between the two groups were tested by the $\chi^{2}$ test or Fisher's exact test, when appropriate. Differences in the continuous variables were compared by a Student's $t$-test or the Wilcoxon rank-sum test when appropriate. Pearson correlation coefficients and the associated probabilities were calculated to assess the relationship between the clinical or biological parameters and the serum androgen levels. All statistical analyses were performed by the R software package (www.R-project.org). All tests were two sided and statistical significance was set at $5 \%$.

\section{Results}

One hundred and three obese adolescent girls and 113 adolescent girls with T1DM met the inclusion and exclusion criteria. The final number of study girls was 174 because seven obese girls and 35 girls with T1DM refused to participate in the study. Table 1 summarises the demography, clinical features and pubertal development of the 96 obese girls and the 78 adolescent girls with T1DM who participated in the study.

\section{Comparison of the obese adolescent girls and adolescent girls with T1DM}

Pubarche, thelarche and menarche occurred significantly earlier in the obese girls than in the girls with T1DM. No differences in the duration of the menstrual cycle and menses were found in the obese girls and the girls with T1DM (Table 1). Puberty was delayed in one obese girl and in six girls with T1DM (1 vs $7.7 \%$ respectively; $P<0.05$ ).

The prevalence of hirsutism and acanthosis was significantly higher in the obese girls than that found in 
Table 1 Demography, pubertal development and clinical laboratory characteristics of the 174 female study participants. Data are displayed as either the median (min - max) or mean \pm S.D.

\begin{tabular}{lll}
\hline & Obesity & T1DM \\
\hline $\begin{array}{l}\text { Number of study participants } \\
\text { Ethnic origin } n \text { (\%) }\end{array}$ & 96 & 78 \\
$\quad$ European Caucasian & $32(33)$ & $38(49)$ \\
North African Caucasian & $25(26)$ & $22(28)$ \\
Black African & $12(12.5)$ & $3(4)$ \\
Other & $27(28)$ & $15(19)$ \\
Age (years) & $14 \pm 1.4$ & $14.3 \pm 1.5$ \\
Age at pubarche (years) & $10.6 \pm 1.3^{*}$ & $11 \pm 1.4$ \\
Age at thelarche (years) & $10 \pm 1.1^{\dagger}$ & $11.4 \pm 1.2$ \\
Age at menarche (years) & $11.9 \pm 1.2^{\dagger}$ & $12.8 \pm 1.2$ \\
Duration of menstrual cycle & $30(15-150)$ & $30(20-120)$ \\
(days) & $5(1-10)$ & $5(3-10)$ \\
Menses duration (days) & $3.8 \pm 0.8$ & $0.8 \pm 1$ \\
BMI (Z-score) & - & $1.09(0.3-1.6)$ \\
Insulin dose (IU/kg per day) & $5.4(4.7-6)$ & $9.1(6.2-13.4)$ \\
Serum HbA1c levels (\%) & $4.4(1.3-37.6)$ & - \\
HOMA-IR & $97.2 \pm 65.3$ & - \\
HOMA\%B &
\end{tabular}

T1DM, type 1 diabetes mellitus; BMI, body mass index; HOMA-IR, homeostatic model assessment of insulin resistance; $\mathrm{HOMA} \% \mathrm{~B}$, homeostatic model assessment for $\beta$-cell function. ${ }^{*} P<0.01,{ }^{\dagger} P<0.001$ and is the significance of the difference between the values of the study parameter from the obese girls and those from girls with T1DM.

the girls with T1DM (hirsutism: 36.5 vs 20.8\%; $P<0.03$, and acanthosis: 51 vs $4 \%$; $P<0.001)$. The Ferriman-Gallwey score of the girls with T1DM and hirsutism was higher than that of obese girls with hirsutism (13.4 \pm 4.3 vs $10.6 \pm 2.9 ; P<0.017)$. The prevalence of menstrual disorders was similar in the obese girls and the girls with T1DM (42 vs 44\%; $P<0.9$ ).

The FAI of the obese girls and T1DM girls with either hirsutism or a menstrual disorder was not significantly different from each other $(P=0.53$ for the girls with hirsutism and $P=0.58$ for the girls with a menstrual disorder) (Tables 2 and 3). However, the serum TT, A and SHBG levels of the girls with T1DM and with either hirsutism or a menstrual disorder were significantly higher than those found in the obese girls with either hirsutism or a menstrual disorder (serum TT levels: $P<0.05$ and $P<0.001$ respectively; serum A levels: $P<0.05$ and $P<0.001$ respectively, and serum SHBG levels $P<0.01$ and $P<0.001$ respectively).

Lastly, the frequency of low serum SHBG levels was greater in obese girls with either hirsutism or a menstrual disorder than that found in T1DM girls with either hirsutism or a menstrual disorder (hirsutism: 49 vs $18.7 \%$; $P=0.06$, and menstrual disorders: 65 vs $20 \% ; P<0.01$ respectively).

\section{Obese adolescent girls}

Obese adolescent girls with hirsutism Hirsutism was found in 35 (36.5\%) of the 96 obese adolescent girls.
The prevalence of hirsutism in the European Caucasian obese girls was significantly lower than that found in the obese girls of other ethnic origins (22 vs 44\%; $P=0.04)$. The mean Ferriman-Gallwey score in obese girls with hirsutism was 10.6 (8-19). The prevalence of T2DM or obesity in first-degree family members of the obese girls with hirsutism was not significantly different from that found in the obese girls without hirsutism (T2DM: 25.7 vs $15 \%$; $P<0.19$ and obesity: 45.7 vs $41.7 \% ; P<0.7)$.

Compared with the obese girls without hirsutism, the obese girls with hirsutism were significantly younger at thelarche and non-significantly younger at pubarche and menarche (Table 2). The prevalence of menstrual disorders in the obese girls with hirsutism was not significantly different from that found in the obese girls without hirsutism $(13 / 29(45 \%)$ in the obese girls with hirsutism vs $7 / 19(37 \%)$ in obese girls without hirsutism; $P=0.58)$.

No statistical significant differences were found for BMI, waist circumference, waist-to-hip ratio, prevalence of acanthosis, blood glucose and plasma insulin levels at OGTT, serum HbA1c levels, the HOMA-IR and the HOMA\%B in these two groups of obese adolescent girls (Table 2). The FAI and the serum TT, A and DHEAS levels in the obese adolescent girls with hirsutism were significantly higher than those found in the obese adolescent girls without hirsutism. The serum SHBG levels in these two groups of obese girls were similar.

Obese adolescent girls with a menstrual disorder Forty-eight of the 96 obese adolescent girls had a gynaecological age of 2 or more years, and of these 48 girls, $20(42 \%)$ had a menstrual disorder. Specifically, ten $(50 \%)$ of these 20 girls had oligomenorrhoea, three (15\%) had polymenorrhoea, six $(30 \%)$ had irregular menstrual cycles and one (5\%) had menorrhagia. The prevalence of T2DM or obesity in first-degree family members of the obese adolescent girls with a menstrual disorder was not significantly different from that found in the obese girls without a menstrual disorder (T2DM: 15 vs $21.4 \%$; $P<0.7$ and obesity: 50 vs $43 \%$; $P<0.6$ ).

The age at puberty of the obese girls with a menstrual disorder was not significantly different from that of the obese girls without a menstrual disorder (Table 2). The duration of obesity in the obese girls with a menstrual disorder was longer than that of the obese girls without a menstrual disorder (11.5 (7.1-13.7) vs 10 (3.6-13.9) years; $P=0.04)$. A similar finding was found for the BMIs and waist circumferences of these two groups of obese girls. The prevalence of hirsutism and acanthosis was not significantly different between these two groups of adolescent girls (Table 2).

The OGTT $30 \mathrm{~min}$ insulin response of the obese girls with a menstrual disorder was significantly greater $(P<0.007)$ than that found in the obese girls without a menstrual disorder. No significant differences in the blood glucose and plasma insulin levels at the other two 
Table 2 Pubertal development and clinical laboratory characteristics of the obese adolescent girls with or without hirsutism or a menstrual disorder. Data are displayed as the median ( $\min -\max$ ). Menstrual disorders were assessed only in girls whose gynaecological age was 2 or more years.

\begin{tabular}{|c|c|c|c|c|c|c|}
\hline & OB H+ & OB H- & $P$ value & OB MD+ & OB MD - & $P$ value \\
\hline$n(\%)$ & $35(36)$ & $61(64)$ & - & $20(42)$ & $28(58)$ & - \\
\hline Age (years) & $14.4(12.2-16.2)$ & $13.9(12-17.9)$ & NS & $15(13.1-16.2)$ & $15(12-17.9)$ & NS \\
\hline Age at pubarche (years) & $10(7-13.6)$ & $10.6(8-13)$ & NS & $10.6(7-12.5)$ & $10(8-12.6)$ & NS \\
\hline Age at thelarche (years) & $10(8-13)$ & $10.6(8-12.6)$ & 0.03 & $10(8-12.5)$ & $10(8-12.6)$ & NS \\
\hline Age at menarche (years) & $11.6(10-14.8)$ & $12.0(9-14.9)$ & NS & $12(10-13.6)$ & $11(9-14)$ & NS \\
\hline BMI (Z-score) & $3.7(2.4-5.8)$ & $3.9(2.3-5.2)$ & NS & $4.2(2.4-5.3)$ & $3.5(2.7-5.8)$ & 0.048 \\
\hline WHR & $0.8(0.8-1.1)$ & $0.9(0.8-1.3)$ & NS & $0.9(0.8-1.2)$ & $0.8(0.8-1)$ & NS \\
\hline Hirsutism (\%) & & & & $7(37)$ & $12(63)$ & NS \\
\hline Acanthosis (\%) & $21(60)$ & $28(46)$ & NS & $10(50)$ & $12(43)$ & NS \\
\hline $\mathrm{FPG}(\mathrm{mmol} / \mathrm{l})$ & $4.7(3.8-6.5)$ & $4.6(3.9-5.6)$ & NS & $4.7(4-5.7)$ & $4.7(3.8-6.5)$ & NS \\
\hline $\mathrm{FPI}(\mathrm{mlU} / \mathrm{l})$ & $21.9(7.9-130)$ & $20.7(7.2-123)$ & NS & $23.9(9-55)$ & $17.7(7.6-130)$ & NS \\
\hline $120 \mathrm{~min} P G$ at OGTT $(\mathrm{mmol} / \mathrm{l})$ & $5.6(3.2-8.2)$ & $5.5(3.6-7.8)$ & NS & $5.5(4.2-8.2)$ & $5.5(3.2-8.2)$ & NS \\
\hline $30 \mathrm{~min} \mathrm{PI}$ at OGTT $(\mathrm{mU} / \mathrm{l})$ & $127(22-397)$ & $115(18-341)$ & NS & $164(41-290)$ & $94(22-313)$ & 0.007 \\
\hline Serum HbA1c (\%) & $5.3(4.7-6)$ & $5.4(4.7-5.9)$ & NS & $5.3(4.7-6)$ & $5.3(4.7-5.7)$ & NS \\
\hline HOMA\%B (\%) & $87.4(38.1-397)$ & $85.4(24.2-478.9)$ & NS & $94(38.4-196.5)$ & $69(28.8-396.5)$ & NS \\
\hline Serum DHEAS (ng/ml) & $1924(746-4935)$ & $1271(243-3704)$ & $<0.001$ & $1819(387-4935)$ & $2031(243-3729)$ & NS \\
\hline Serum A (ng/ml) & $1.8(0.3-4.1)$ & $1.4(0.6-3)$ & 0.053 & $1.8(0.8-3)$ & $1.9(0.6-4.1)$ & NS \\
\hline Serum TT (ng/ml) & $0.4(0.1-1)$ & $0.3(0.1-0.9)$ & 0.004 & $0.4(0.1-0.9)$ & $0.3(0.1-1)$ & NS \\
\hline Serum SHBG (nmol/l) & $26.4(4-86)$ & $24.5(7.7-112)$ & NS & $20(4-43)$ & $25.4(10-76)$ & NS \\
\hline $\mathrm{FAI}(\mathrm{nmol} / \mathrm{nmol}$ per I) & $5.7(0.7-40)$ & $2.6(0.8-8.6)$ & 0.004 & $5.7(1-39.9)$ & $4.3(0.8-11.5)$ & NS \\
\hline
\end{tabular}

T1DM, type 1 diabetes mellitus; $\mathrm{OB}$, obesity; $\mathrm{H}$, hirsutism; $\mathrm{H}+$, with hirsutism; $\mathrm{H}-$, without hirsutism; MD, menstrual disorder; MD+, with a menstrual disorder; MD -, without a menstrual disorder; BMI, body mass index; WC, waist circumference; WHR, waist-to-hip ratio; FPG, fasting plasma glucose; FPI, fasting plasma insulin; PG, plasma glucose; PI, plasma insulin; OGTT, oral glucose tolerance test; HOMA-IR, homeostasis model assessment of insulin resistance; $\mathrm{HOMA} \% \mathrm{~B}$, homeostatic model assessment for $\beta$-cell function; $\mathrm{A}$, androstenedione; TT, total testosterone; SHBG, sex hormone-binding globulin; FAl, free androgen index; NS, not significant.

time points of the OGTT, the serum HbA1c levels and the indices of insulin secretion and sensitivity were found in these two groups of obese girls (Table 2). The serum androgen levels in these two groups of obese girls were similar. Serum SHBG levels were decreased to the same extent in these two groups. The FAI of the obese girls with a menstrual disorder was not significantly different from that found in the obese girls without a menstrual disorder.

Relationship between FAI and the study parameters of the obese adolescent girls Waist circumference was significantly correlated $\left(R^{2}=0.35 ; P<0.01\right)$ with the FAI of the obese girls. Neither the serum HbA1c levels nor the two insulin indices were significantly correlated with the serum androgen levels.

\section{Adolescent girls with T1DM}

Adolescent girls with T1DM and hirsutism Hirsutism was found in 16 (21\%) of the 78 girls with T1DM. Although the prevalence of hirsutism in the European Caucasian girls with T1DM was lower than that of the T1DM girls of other ethnic origins, this difference was not statistically significant (13 vs $28 \% ; P=0.11)$. The mean Ferriman-Gallwey score in the girls with T1DM and hirsutism was 13.4 (8-22). The prevalence of T2DM and obesity in first-degree family members of the girls with T1DM and hirsutism was higher, but not statistically significant, than that of the girls with T1DM and without hirsutism (T2DM: 33 vs $11.7 \%$; $P>0.05$ and obesity: 20 vs $6 \% ; P<0.1)$.

The girls with T1DM and hirsutism were significantly older than the girls with T1DM and without hirsutism. No statistically significant differences were found for the ages at pubarche, thelarche and menarche and the prevalence of menstrual disorders for these two groups of T1DM girls (62\% in girls with T1DM and hirsutism vs $30 \%$ in girls with T1DM and without hirsutism; $P=0.07)$.

Among the girls with T1DM, the waist circumferences of those with hirsutism were significantly higher than those without hirsutism, whereas their BMI values were similar. The duration of the disease in the girls with T1DM and hirsutism was longer than that of the girls with T1DM and without hirsutism (6.7 (1.9-14.5) vs $4.8(0.4-12.6)$ years; $P=0.02)$, and the girls with T1DM and hirsutism had non-significant higher daily insulin requirements and serum HbA1c levels than the girls with T1DM and without hirsutism. The FAI, and serum A and DHEAS levels of the girls with T1DM and hirsutism were significantly higher than those found in the girls with T1DM and without hirsutism. No significant differences were found in the serum TT and SHBG levels of these two groups of girls with T1DM (Table 3). 
Table 3 Pubertal development and clinical laboratory characteristics of the adolescent girls with T1DM with or without hirsutism or a menstrual disorder. Data are displayed as median ( $\min -\max$ ). Menstrual disorders were assessed only in girls whose gynaecological age was two or more years.

\begin{tabular}{|c|c|c|c|c|c|c|}
\hline & T1DM H+ & T1DM H- & $P$ value & T1DM MD+ & T1DM MD - & $P$ value \\
\hline$n(\%)$ & $16(21)$ & $62(79)$ & - & $15(44)$ & $19(56)$ & \\
\hline Age (years) & $15.5(13-17.4)$ & $14(11.9-17.5)$ & $<0.001$ & $15.5(13.9-17.4)$ & $14.7(11.9-17.5)$ & 0.11 \\
\hline Age at pubarche (years) & $10.6(8-14)$ & $11(8-14)$ & NS & $10.6(8-14)$ & $10.5(8-13)$ & NS \\
\hline Age at thelarche (years) & $11.3(8.6-13)$ & $11.3(8-13.8)$ & NS & $11(8-13)$ & $10.5(8-12)$ & NS \\
\hline Age at menarche (years) & $13(11-14)$ & $13(10-15.3)$ & NS & $13(11-14)$ & $12(10-14.5)$ & 0.02 \\
\hline BMI (Z-score) & $1.1(-0.7-3.7)$ & $0.8(-2.1-2)$ & NS & $1.06(-0.7-2)$ & $1.3(0.4-2.9)$ & NS \\
\hline WC $(\mathrm{cm})$ & $77(69-90)$ & $70(61-85)$ & $<0.001$ & $75.5(69-88)$ & $73(70-85)$ & NS \\
\hline WHR & $0.8(0.7-1)$ & $0.8(0.7-1.8)$ & NS & $0.8(0.7-1)$ & $0.8(0.7-0.9)$ & NS \\
\hline Hirsutism (\%) & & & & $8(62)$ & $5(39)$ & NS \\
\hline Insulin dose (IU/kg per day) & $1.1(0.7-1.3)$ & $1(0.3-1.6)$ & NS & $1(0.3-1.5)$ & $1.1(0.6-1.6)$ & NS \\
\hline Serum $\mathrm{HbA} 1 \mathrm{c}(\%)$ & $9.6(6.2-12.2)$ & $8.6(6.7-13.4)$ & NS & $9.3(6.2-13.4)$ & $8.6(6.7-13.3)$ & NS \\
\hline Serum DHEAS (ng/ml) & $1978(622-5196)$ & $1004(208-2729)$ & $<0.001$ & $1931(765-4116)$ & $1709(722-5196)$ & NS \\
\hline Serum A $(\mathrm{ng} / \mathrm{ml})$ & $2.6(1.1-6.7)$ & $1.9(0.7-3.8)$ & 0.03 & $2.9(1.6-5.7)$ & $1.9(1.1-3.6)$ & 0.001 \\
\hline Serum TT $(\mathrm{ng} / \mathrm{ml})$ & $0.6(0.2-1.4)$ & $0.5(0.1-1.1)$ & 0.29 & $0.7(0.2-1.4)$ & $0.5(0.2-1)$ & 0.01 \\
\hline Serum SHBG $(\mathrm{nmol} / \mathrm{l})$ & $47(16-92)$ & $52.5(15-115)$ & 0.19 & $43(15-59)$ & $47(24-81)$ & NS \\
\hline FAl & $5.8(1-23)$ & $2.9(0.8-14.9)$ & 0.04 & $6.5(2.1-23.1)$ & $3.6(1.1-14.9)$ & 0.01 \\
\hline
\end{tabular}

T1DM, type 1 diabetes mellitus; $\mathrm{H}$, hirsutism; $\mathrm{H}+$, with hirsutism; $\mathrm{H}-$, without hirsutism; MD, menstrual disorder; MD +, with a menstrual disorder; MD -, without a menstrual disorder; BMI, body mass index; WC, waist circumference; WHR, waist-to-hip ratio; A, androstenedione; TT, total testosterone; SHBG, sex hormone-binding globulin; FAI, free androgen index; NS, not significant.

\begin{abstract}
Adolescent girls with T1DM and a menstrual disorder Thirty-four of the 78 girls with T1DM had gynaecological ages of 2 or more years, and of these 34 girls, $15(44 \%)$ had a menstrual disorder. Specifically, nine $(60 \%)$ of these 15 girls had oligomenorrhoea, one (6\%) had secondary amenorrhoea and five (33\%) had irregular menstrual cycles. The prevalence of T2DM or obesity in first-degree family members of girls with T1DM and a menstrual disorder was not significantly different from those girls with T1DM and without a menstrual disorder (T2DM: 28.6 vs $15.8 \% ; P=0.42$ and obesity: 21.4 vs $10.5 \% ; P=0.62$ ). Although menarche in the girls with T1DM and a menstrual disorder occurred later than in the girls with T1DM and without a menstrual disorder, no significant differences were found in the age at pubarche or thelarche in these two groups of girls with T1DM.
\end{abstract}

Among the girls with T1DM, no significant differences in BMI, waist circunference, waist-to-hip ratio, prevalence of hirsutism, duration of diabetes mellitus, daily insulin dose and serum $\mathrm{HbAlc}$ levels were found between those with and without a menstrual disorder. The FAI and the serum TT and A levels in the girls with T1DM and a menstrual disorder were significantly higher than those found in girls with T1DM and without a menstrual disorder. No significant differences were found in the serum DHEAS and SHBG levels in these two groups of girls with T1DM (Table 3).

\section{Relationship between FAI and the study parameters in girls with T1DM The FAI was significantly correlated with their BMI $\left(R^{2}=0.36 ; P<0.01\right)$, their waist circumference $\left(R^{2}=0.50 ; P<0.001\right)$ and their serum HbA1c levels $\left(R^{2}=0.44 ; P<0.001\right)$.}

\section{Discussion}

The purpose of this study was to evaluate and compare the prevalence of hirsutism or a menstrual disorder, the pubertal development and the hormonal profiles of obese adolescent girls and adolescent girls with T1DM. Although a few studies have investigated these characteristics in obese adolescent girls and adolescent girls with T1DM, to the best of our knowledge, this is the first investigation that has compared the clinical and biological characteristics of hirsutism or menstrual disorders in these two groups of adolescent girls.

We found that pubertal development in the obese adolescent girls occurred earlier than that in the adolescent girls with T1DM. This finding is in agreement with what has been reported by others in obese (34) and adolescent girls with T1DM $(35,36,37,38)$.

We found that the prevalence of hirsutism in our cohort of obese adolescent girls was $36.5 \%$, which is higher than that found in healthy women $(2-8 \%)(39$, $40)$ and adolescent girls (6\%) (41). To the best of our knowledge, there are no reports on the prevalence of hirsutism in obese adolescent girls independently of the presence of polycystic ovary syndrome. We also found menstrual disorders in $42 \%$ of the obese adolescent girls. This prevalence was higher than that previously reported in obese adolescents $(27.2 \%)(42)$, in obese women $(26 \%$ (43), 30\% (44) and $35.7 \%(45))$ and in healthy French girls (20\%) (unpublished data from an epidemiological study of the Institut Inter Regional Pour La Santé).

The results of previous investigations reported the existence of a relationship among the degree of obesity, hyperinsulinism, the decline in serum SHBG levels and hyperandrogenism in obese girls and women $(2,12,14$, 
$42,43,46)$. This finding is at variance with the results of our study because the obese girls with hirsutism, despite their hyperandrogenism that correlated with their waist circumference, did not have a greater degree of obesity, higher plasma insulin levels or lower serum SHBG levels than the obese girls without hirsutism. In accordance with our findings, menstrual disorders in obese women have been associated with a high degree and early onset of obesity, centrally distributed body fat $(12,14,43,47)$ and a high $30 \mathrm{~min}$ insulin peak in the OGTT (48). We found no significant differences in the serum androgen levels of the obese adolescent girls with a menstrual disorder than those without a menstrual disorder. This finding of no difference could be due to i) the small sample of adolescent girls in each of these study subgroups or ii) factors other than hyperandrogenism, such as failure of the normal feedforward and feedback signaling between the pituitary and ovaries. Indeed, Yoo et al. (49) found increased LH pulse frequency in obese girls with oligomenorrhoea in the absence of clinical and/or biochemical evidence of hyperandrogenism.

The prevalence of hirsutism in our cohort of adolescent girls with T1DM was $21 \%$. Since we are not aware of other published reports on the prevalence of hirsutism in adolescent girls with T1DM, we are unable to make a compare-and-contrast analysis of our finding. However, we can comment that our finding of the $21 \%$ prevalence of hirsutism in our cohort of adolescent girls with T1DM is markedly higher than the $7.1 \%$ prevalence of hirsutism in non-diabetic women (39) and almost as high as the $30 \%$ prevalence of hirsutism in T1DM women that was reported by Escobar-Morreale et al. (5) and Codner \& EscobarMorreale (19).

We found that the prevalence of a menstrual disorder in the adolescent girls with T1DM was high $(44 \%)$. This value of prevalence was higher than that previously reported by Schroeder et al. (18) (19\%), but almost as high as that reported by Adcock et al. $(15,50)(54 \%)$ and Gaete et al. $(15,50)(58.9 \%)$ in adolescent girls with T1DM.

The results of previous investigations have found that a high BMI, large insulin doses, a high serum HbAlc level, a large LH/FSH ratio and a low SHBG level are all associated with menstrual irregularities in adolescent girls with T1DM $(15,18,50)$. However, Gaete et al. (15) reported that the prevalence of oligomenorrhoea in girls with T1DM whose serum HbAlc levels below $7.6 \%$ is similar to that found in girls with T1DM, whose serum $\mathrm{HbA1c}$ levels are greater than 9\%. In the adolescent girls with T1DM, we found that their glycaemic control was correlated with their hyperandrogenism. The intensive insulin therapy, which is usually needed to achieve good glycaemic control in T1DM women, may result in high circulating insulin levels. These increased circulating levels result in increased insulin exposure of the hypothalamus, ovaries and adrenal glands, which could promote the development of a menstrual disorder in these women $(4,19,20,51)$. However, in our cohort of adolescent girls with T1DM, we found no significant differences in the daily insulin doses that were given to the girls with and without hirsutism or the girls who complained of a menstrual disorder. In keeping with these results, a previous study of women with T1DM detected no significant differences in the insulin dosage or the glycaemic control of these women with or without hyperandrogenism (5). The only clinical characteristic that we found to be significantly different was the waist circumference of the adolescent girls with T1DM and hirsutism compared with that measured in those adolescent girls with T1DM and without hirsutism. This difference in waist circumference may be due to the high insulin resistance, which is frequently found in adolescent girls with T1DM, and the secondary hyperinsulinaemia, which is associated with a gain in fat mass throughout puberty in such girls (52).

In our study, we found the hyperandrogenic girls with T1DM had high FAI and high serum A levels, but normal serum SHBG levels. Roldan et al. (20) reported that hyperandrogenic women with T1DM have increased serum levels of free testosterone and $\mathrm{A}$, and normal serum SHBG levels. They also reported that these women have normal serum DHEAS levels after the ACTH stimulation test. Their findings suggest that the contribution of the adrenal gland secretions to the androgen excess of these women is small. Nonetheless, we found high serum DHEAS levels in adolescent girls with T1DM and hirsutism. Our result could mean that the adrenal gland secretions may contribute to the development of hyperandrogenism in girls with T1DM.

We also investigated the mechanism of hyperandrogenism in obese adolescent girls and adolescent girls with T1DM. We found that FAI values were similar in the two groups of girls with either hirsutism or a menstrual disorder, but their androgen profiles differed. Specifically, the serum androgen levels were higher in the adolescent girls with T1DM than in the obese adolescent girls. We speculate that the difference in the androgen profiles could be due to different sources of the excess androgens. In the obese girls, insulin resistance and hyperinsulinaemia with increased intraportal insulin levels inhibit hepatic SHBG production, thereby increasing the FAI $(2,12,46,53)$. In the girls with T1DM, subcutaneously administered insulin is associated with increased peripheral bioavailability of insulin and low intraportal insulin levels and reduced inhibition of hepatic SHBG production (4, 15, 19). Although serum androgen levels in the adolescent girls with T1DM were higher than those in the obese adolescent, hirsutism was more common in the obese girls. We posit that the prevalence of hirsutism in the obese girls is due to their increased androgen bioavailability and their low serum SHBG levels.

A limitation of the study could be the fact that no control group was included. Such a group could not be incorporated into the study because venous blood 
sampling in healthy children was not deemed ethical by the institutional review board. Furthermore, the daily insulin doses that were reported by the adolescent girls with T1DM are a subjective parameter, and may have been overestimated or underestimated by the girls. We were also unable to establish the existence of an association between those factors that are usually associated with hyperandrogenism in obese girls, namely the degree of obesity and hyperinsulinaemia and hyperandrogenism in girls with T1DM, namely BMI, insulin dose and glycaemic control. The reason for our inability to establish such an existence was the small number of each study cohort, which prevented us from doing linear regression analysis.

The results of this study confirm that puberty occurs earlier in obese adolescent girls than those with T1DM. The results also show that the prevalence of hirsutism and menstrual disorders is high in obese adolescent girls and adolescent girls with T1DM. Although the serum free testosterone levels are elevated in these two groups of adolescent girls, their androgenic profiles differ. The obese girls showed hyperandrogenaemia, which is mostly secondary to their decreased serum SHBG levels, whereas the girls with T1DM have increased androgen production. Thus, routine evaluations for hirsutism and menstrual disorders in adolescent girls and adolescent girls with T1DM must be done regularly. The follow-up of such girls should include a complete record of their menstrual history, a physical examination and FAI determination at regular intervals.

\section{Declaration of interest}

The authors confirm that the manuscript or any part of it, except for presentation of an abstract at a medical science conference, has not been published or is being considered for publication elsewhere. They also declare that there is no conflict of interest that could be perceived as prejudicing the impartiality of the reported research.

\section{Funding}

This work was financially supported by the Wyeth Foundation and in part by the 'Association des Jeunes Diabétiques' (AJD). A Colmenares was financially supported by Fundayacucho, Venezuela.

\section{Acknowledgements}

We are deeply indebted to the adolescent girls who agreed to participate in this study, and the nursing staff at Necker Children's Hospital and the Clinical Investigation Centre at the Robert Debré Hospital. The authors also thank Dr Arieh Bomzon, Consulwrite (www.consulwrite.com) for his editorial assistance in preparing this manuscript.

\section{References}

1 Mauras N, Welch S, Rini A \& Haymond MW. Ovarian hyperandrogenism is associated with insulin resistance to both peripheral carbohydrate and whole-body protein metabolism in postpubertal young females: a metabolic study. Journal of Clinical Endocrinology and Metabolism 199883 1900-1905. (doi:10. 1210/jc.83.6.1900)

2 McCartney CR, Prendergast KA, Chhabra S, Eagleson CA, Yoo R, Chang RJ, Foster CM \& Marshall JC. The association of obesity and hyperandrogenemia during the pubertal transition in girls: obesity as a potential factor in the genesis of postpubertal hyperandrogenism. Journal of Clinical Endocrinology and Metabolism 200691 1714-1722. (doi:10.1210/jc.2005-1852)

3 Reinehr T, de Sousa G, Roth CL \& Andler W. Androgens before and after weight loss in obese children. Journal of Clinical Endocrinology and Metabolism 200590 5588-5595. (doi:10.1210/jc.20050438)

4 Codner E \& Cassorla F. Puberty and ovarian function in girls with type 1 diabetes mellitus. Hormone Research 2009 71 12-21. (doi:10.1159/000173737)

5 Escobar-Morreale HF, Roldan B, Barrio R, Alonso M, Sancho J, de la Calle H \& Garcia-Robles R. High prevalence of the polycystic ovary syndrome and hirsutism in women with type 1 diabetes mellitus. Journal of Clinical Endocrinology and Metabolism 200085 4182-4187. (doi:10.1210/jc.85.11.4182)

6 Willis D, Mason H, Gilling-Smith C \& Franks S. Modulation by insulin of follicle-stimulating hormone and luteinizing hormone actions in human granulosa cells of normal and polycystic ovaries. Journal of Clinical Endocrinology and Metabolism 199681 302-309. (doi:10.1210/jc.81.1.302)

7 Rosenfield RL. Evidence that idiopathic functional adrenal hyperandrogenism is caused by dysregulation of adrenal steroidogenesis and that hyperinsulinemia may be involved. Journal of Clinical Endocrinology and Metabolism 199681 878-880. (doi:10. $1210 /$ jc.81.3.878)

8 Nestler JE \& Jakubowicz DJ. Decreases in ovarian cytochrome P450c17 alpha activity and serum free testosterone after reduction of insulin secretion in polycystic ovary syndrome. New England Journal of Medicine 1996335 617-623. (doi:10.1056/ NEJM199608293350902)

9 Nestler JE, Jakubowicz DJ, de Vargas AF, Brik C, Quintero N \& Medina F. Insulin stimulates testosterone biosynthesis by human thecal cells from women with polycystic ovary syndrome by activating its own receptor and using inositolglycan mediators as the signal transduction system. Journal of Clinical Endocrinology and Metabolism 199883 2001-2005. (doi:10.1210/jc.83.6.2001)

10 Nestler JE, Clore JN, Strauss JF III \& Blackard WG. The effects of hyperinsulinemia on serum testosterone, progesterone, dehydroepiandrosterone sulfate, and cortisol levels in normal women and in a woman with hyperandrogenism, insulin resistance, and acanthosis nigricans. Journal of Clinical Endocrinology and Metabolism 198764 180-184. (doi:10.1210/jcem-64-1-180)

11 Munir I, Yen HW, Geller DH, Torbati D, Bierden RM, Weitsman SR, Agarwal SK \& Magoffin DA. Insulin augmentation of 17alphahydroxylase activity is mediated by phosphatidyl inositol 3-kinase but not extracellular signal-regulated kinase-1/2 in human ovarian theca cells. Endocrinology 2004145 175-183. (doi:10. 1210/en.2003-0329)

12 Pasquali R \& Gambineri A. Metabolic effects of obesity on reproduction. Reproductive Biomedicine Online $200612542-$ 551. (doi:10.1016/S1472-6483(10)61179-0)

13 Strotmeyer ES, Steenkiste AR, Foley TP Jr, Berga SL \& Dorman JS. Menstrual cycle differences between women with type 1 diabetes and women without diabetes. Diabetes Care 200326 1016-1021. (doi:10.2337/diacare.26.4.1016)

14 Gambineri A, Pelusi C, Vicennati V, Pagotto U \& Pasquali R. Obesity and the polycystic ovary syndrome. International Journal of Obesity and Related Metabolic Disorders 200226 883-896. (doi:10. 1038/sj.ijo.0801994)

15 Gaete X, Vivanco M, Eyzaguirre FC, Lopez P, Rhumie HK, Unanue N \& Codner E. Menstrual cycle irregularities and their relationship with $\mathrm{HbA1c}$ and insulin dose in adolescents with type 1 diabetes mellitus. Fertility and Sterility 201094 1822-1826. (doi:10.1016/j.fertnstert.2009.08.039) 
16 Hartz AJ, Barboriak PN, Wong A, Katayama KP \& Rimm AA. The association of obesity with infertility and related menstrual abnormalities in women. International Journal of Obesity 19793 57-73.

17 Meyer K, Deutscher J, Anil M, Berthold A, Bartsch M \& Kiess W. Serum androgen levels in adolescents with type 1 diabetes: relationship to pubertal stage and metabolic control. Journal of Endocrinological Investigation 200023 362-368.

18 Schroeder B, Hertweck SP, Sanfilippo JS \& Foster MB. Correlation between glycemic control and menstruation in diabetic adolescents. Journal of Reproductive Medicine 200045 1-5.

19 Codner E \& Escobar-Morreale HF. Clinical review: hyperandrogenism and polycystic ovary syndrome in women with type 1 diabetes mellitus. Journal of Clinical Endocrinology and Metabolism 200792 1209-1216. (doi:10.1210/jc.2006-2641)

20 Roldan B, Escobar-Morreale HF, Barrio R, de La Calle H, Alonso M, Garcia-Robles R \& Sancho J. Identification of the source of androgen excess in hyperandrogenic type 1 diabetic patients. Diabetes Care 200124 1297-1299. (doi:10.2337/diacare.24.7. 1297)

21 Pietrobelli A, Faith MS, Allison DB, Gallagher D, Chiumello G \& Heymsfield SB. Body mass index as a measure of adiposity among children and adolescents: a validation study. Journal of Pediatrics 1998132 204-210. (doi:10.1016/S0022-3476(98)70433-0)

22 Rolland-Cachera MF, Cole TJ, Sempe M, Tichet J, Rossignol C \& Charraud A. Body mass index variations: centiles from birth to 87 years. European Journal of Clinical Nutrition 199145 13-21.

23 Ferriman D \& Gallwey JD. Clinical assessment of body hair growth in women. Journal of Clinical Endocrinology and Metabolism 1961 21 1440-1447. (doi:10.1210/jcem-21-11-1440)

24 van Hooff MH, Voorhorst FJ, Kaptein MB, Hirasing RA, Koppenaal C \& Schoemaker J. Relationship of the menstrual cycle pattern in 14-17 year old old adolescents with gynaecological age, body mass index and historical parameters. Human Reproduction 199813 2252-2260. (doi:10.1093/humrep/13.8. 2252)

25 van Hooff MH, Voorhorst FJ, Kaptein MB, Hirasing RA, Koppenaal C \& Schoemaker J. Predictive value of menstrual cycle pattern, body mass index, hormone levels and polycystic ovaries at age 15 years for oligo-amenorrhoea at age 18 years. Human Reproduction 200419 383-392. (doi:10.1093/humrep/ deh079)

26 Legro RS, Lin HM, Demers LM \& Lloyd T. Rapid maturation of the reproductive axis during perimenarche independent of body composition. Journal of Clinical Endocrinology and Metabolism 200085 1021-1025. (doi:10.1210/jc.85.3.1021)

27 Adams Hillard PJ. Menstruation in adolescents: what's normal, what's not. Annals of the New York Academy of Sciences 20081135 29-35. (doi:10.1196/annals.1429.022)

28 World Health Organization multicenter study on menstrual and ovulatory patterns in adolescent girls. II. Longitudinal study of menstrual patterns in the early postmenarcheal period, duration of bleeding episodes and menstrual cycles. World Health Organization Task Force on Adolescent Reproductive Health. Journal of Adolescent Health Care 19867 236-244. (doi:10.1016/ S0197-0070(86)80015-8)

29 Lashansky G, Saenger P, Fishman K, Gautier T, Mayes D, Berg G, Di Martino-Nardi J \& Reiter E. Normative data for adrenal steroidogenesis in a healthy pediatric population: age- and sexrelated changes after adrenocorticotropin stimulation. Journal of Clinical Endocrinology and Metabolism 199173 674-686. (doi:10. 1210/jcem-73-3-674)

30 Vermeulen A, Verdonck L \& Kaufman JM. A critical evaluation of simple methods for the estimation of free testosterone in serum. Journal of Clinical Endocrinology and Metabolism 1999843666 3672. (doi:10.1210/jc.84.10.3666)

31 Matthews DR, Hosker JP, Rudenski AS, Naylor BA, Treacher DF \& Turner RC. Homeostasis model assessment: insulin resistance and beta-cell function from fasting plasma glucose and insulin concentrations in man. Diabetologia 198528 412-419. (doi:10. 1007/BF00280883)
32 Wallace TM \& Matthews DR. The assessment of insulin resistance in man. Diabetic Medicine 200219 527-534. (doi:10.1046/j. 1464-5491.2002.00745.x)

33 Wallace TM, Levy JC \& Matthews DR. Use and abuse of HOMA modeling. Diabetes Care 200427 1487-1495. (doi:10.2337/ diacare.27.6.1487)

34 Kaplowitz PB, Slora EJ, Wasserman RC, Pedlow SE \& HermanGiddens ME. Earlier onset of puberty in girls: relation to increased body mass index and race. Pediatrics 2001108 347-353. (doi:10. 1542/peds.108.2.347)

35 Kjaer K, Hagen C, Sando SH \& Eshoj O. Epidemiology of menarche and menstrual disturbances in an unselected group of women with insulin-dependent diabetes mellitus compared to controls. Journal of Clinical Endocrinology and Metabolism $1992 \mathbf{7 5}$ 524-529. (doi:10.1210/jc.75.2.524)

36 Codner E, Barrera A, Mook-Kanamori D, Bazaes RA, Unanue N, Gaete X, Avila A, Ugarte F, Torrealba I, Perez V, Panteon E \& Cassorla F. Ponderal gain, waist-to-hip ratio, and pubertal development in girls with type-1 diabetes mellitus. Pediatric Diabetes 20045 182-189. (doi:10.1111/j.1399-543X.2004. 00059.x)

37 Danielson KK, Palta M, Allen C \& D'Alessio DJ. The association of increased total glycosylated hemoglobin levels with delayed age at menarche in young women with type 1 diabetes. Journal of Clinical Endocrinology and Metabolism 200590 6466-6471. (doi:10. 1210/jc.2005-0349)

38 Picardi A, Cipponeri E, Bizzarri C, Fallucca S, Guglielmi C \& Pozzilli P. Menarche in type 1 diabetes is still delayed despite good metabolic control. Fertility and Sterility 200890 1875-1877. (doi:10.1016/j.fertnstert.2007.09.035)

39 Asuncion M, Calvo RM, San Millan JL, Sancho J, Avila S \& EscobarMorreale HF. A prospective study of the prevalence of the polycystic ovary syndrome in unselected Caucasian women from Spain. Journal of Clinical Endocrinology and Metabolism $2000 \mathbf{8 5}$ 2434-2438. (doi:10.1210/jc.85.7.2434)

40 Knochenhauer ES, Key TJ, Kahsar-Miller M, Waggoner W, Boots LR \& Azziz R. Prevalence of the polycystic ovary syndrome in unselected black and white women of the southeastern United States: a prospective study. Journal of Clinical Endocrinology and Metabolism $1998 \mathbf{8 3}$ 3078-3082. (doi:10. 1210/jc.83.9.3078)

41 Hashemipour M, Faghihimani S, Zolfaghary B, Hovsepian S, Ahmadi F \& Haghighi S. Prevalence of polycystic ovary syndrome in girls aged 14-18 years in Isfahan, Iran. Hormone Research 2004 62 278-282. (doi:10.1159/000081842)

42 Alemzadeh R, Kichler J \& Calhoun M. Spectrum of metabolic dysfunction in relationship with hyperandrogenemia in obese adolescent girls with polycystic ovary syndrome. European Journal of Endocrinology 2010162 1093-1099. (doi:10.1530/ EJE-10-0205)

43 Wei S, Schmidt MD, Dwyer T, Norman RJ \& Venn AJ. Obesity and menstrual irregularity: associations with SHBG, testosterone, and insulin. Obesity 200917 1070-1076. (doi:10.1038/oby.2008. 641)

44 Castillo-Martinez L, Lopez-Alvarenga JC, Villa AR \& GonzalezBarranco J. Menstrual cycle length disorders in 18- to 40-y-old obese women. Nutrition $200319317-320$. (doi:10.1016/S08999007(02)00998-X)

45 De Pergola G, Tartagni M, d'Angelo F, Centoducati C, Guida P \& Giorgino R. Abdominal fat accumulation, and not insulin resistance, is associated to oligomenorrhea in non-hyperandrogenic overweight/obese women. Journal of Endocrinological Investigation 200932 98-101.

46 De Simone M, Verrotti A, Iughetti L, Palumbo M, Farello G, Di Cesare E, Bernabei R, Rosato T, Lozzi S \& Criscione S. Increased visceral adipose tissue is associated with increased circulating insulin and decreased sex hormone binding globulin levels in massively obese adolescent girls. Journal of Endocrinological Investigation $2001 \mathbf{2 4} 438-444$. 
47 Douchi T, Kuwahata R, Yamamoto S, Oki T, Yamasaki H \& Nagata Y. Relationship of upper body obesity to menstrual disorders. Acta Obstetricia et Gynecologica Scandinavica 200281 147-150. (doi:10.1034/j.1600-0412.2002.810210.x)

48 Morrison JA, Glueck CJ, Daniels S, Wang P \& Stroop D. Ramifications of adolescent menstrual cycles $>/=42$ days in young adults. Fertility and Sterility 201196 236.e1-240.e1. (doi:10.1016/j.fertnstert.2011.04.005)

49 Yoo RY, Dewan A, Basu R, Newfield R, Gottschalk M \& Chang RJ. Increased luteinizing hormone pulse frequency in obese oligomenorrheic girls with no evidence of hyperandrogenism. Fertility and Sterility $2006 \mathbf{8 5}$ 1049-1056. (doi:10.1016/j.fertnstert.2005. 09.037)

50 Adcock CJ, Perry LA, Lindsell DR, Taylor AM, Holly JM, Jones J \& Dunger DB. Menstrual irregularities are more common in adolescents with type 1 diabetes: association with poor glycaemic control and weight gain. Diabetic Medicine 199411 465-470. (doi:10.1111/j.1464-5491.1994.tb00307.x)

51 Bruning JC, Gautam D, Burks DJ, Gillette J, Schubert M, Orban PC, Klein R, Krone W, Muller-Wieland D \& Kahn CR. Role of brain insulin receptor in control of body weight and reproduction. Science 2000289 2122-2125. (doi:10.1126/science.289.5487. 2122)

52 Ahmed ML, Ong KK, Watts AP, Morrell DJ, Preece MA \& Dunger DB. Elevated leptin levels are associated with excess gains in fat mass in girls, but not boys, with type 1 diabetes: longitudinal study during adolescence. Journal of Clinical Endocrinology and Metabolism 2001 86 1188-1193. (doi:10.1210/jc.86.3.1188)

53 Yki-Jarvinen H, Makimattila S, Utriainen T \& Rutanen EM. Portal insulin concentrations rather than insulin sensitivity regulate serum sex hormone-binding globulin and insulin-like growth factor binding protein 1 in vivo. Journal of Clinical Endocrinology and Metabolism $1995 \mathbf{8 0}$ 3227-3232. (doi:10. 1210/jc.80.11.3227)

Received 15 March 2011

Revised version received 12 November 2011

Accepted 28 November 2011 